\title{
The nature of the nearest compact group of galaxies from precise distance measurements (Research Note)
}

\author{
G. A. Mamon \\ Institut d'Astrophysique de Paris (UMR 7095: CNRS \& UPMC), 98 bis Bd Arago, 75014 Paris, France \\ e-mail: gam@iap.fr
}

Received 21 March 2008 / Accepted 13 April 2008

\begin{abstract}
Context. Compact groups (CGs) of galaxies, similar to those catalogued by Hickson, appear to be the densest galaxy structures in the Universe. Redshift information is insufficient to determine whether a CG is roughly as dense in three dimensions as it appears in projection, or whether it is caused by a chance alignment along the line of sight within a larger galaxy system.

Aims. Recent precise distance measurements help probe the nature of the nearest CG, situated in the Virgo cluster, whose dominant member is M 60.

Methods. The isolated status of the CG is reassessed with recent photometry and a statistical analysis is performed on the surface brightness fluctuation (SBF) distances measured by Mei et al. in Virgo, for 4 of the 5 CG members.

Results. The neighboring galaxy NGC 4606 appears (with 80-90\% confidence) to be too faint to affect the isolated status of the CG. Taken at face value, the SBF distances suggest that M 59 and NGC 4660 lie roughly 2 Mpc to the foreground of M 60, NGC 4638, and the bulk of the Virgo cluster. The statistical analysis confirms that the CG is, indeed, the result of a chance alignment of its galaxies, with NGC 4638 lying at least $800 \mathrm{kpc}$ further away (with 99\% confidence) than either M 59 or NGC 4660. The first two galaxy distances are consistent with M 59 and NGC 4660 constituting a tight pair. The dominant galaxy, M 60, is at least $440 \mathrm{kpc}$ more distant (95\% confidence) than the M 59+NGC 4660 pair, and over 1 Mpc (99\% confidence) more distant if one uses the broken linear calibration of the SBF distances.

Conclusions. This work constitutes the first direct analysis of the nature of a compact group of galaxies. Chance alignments of galaxies represent a realistic alternative to truly dense groups to explain the nature of CGs. With current SBF distance accuracies, one could determine the nature of HCG 68 in the same way.
\end{abstract}

Key words. galaxies: clusters: individual: Virgo - galaxies: distances and redshifts

\section{Introduction}

Compact groups of galaxies (CGs) appear to be the densest known multiple galaxy systems (with mean densities $\sim 10^{5}$ times the critical density of the Universe). The CG catalog by far the most studied is the one assembled by Hickson (1982), who visually searched the POSS I photographic plates for isolated groups of at least 4 members within 3 mag of the brightest, whose mean surface brightness exceeded a given threshold. The mean surface brightness is measured within the smallest circumscribed circle (hereafter, Hickson circle) containing the centers of the galaxies. The isolation criterion specifies that there are no galaxies within 3 mag (in the $R$ band) from the brightest CG member within an isolation ring extending from the Hickson circle to a concentric circle 3 times wider.

A spectroscopic followup by Hickson et al. (1992) revealed that among the 100 Hickson compact groups (HCGs), only 69 groups had at least four members with accordant velocities (within $1000 \mathrm{~km} \mathrm{~s}^{-1}$ from the median). Still, it is unclear whether these 69 HCGs are roughly as dense in three dimensions as they appear to be in projection (Hickson \& Rood 1988), or whether they are caused by chance alignments of galaxies along the line of sight (Rose 1977, for the elongated CGs; Mamon 1986 and Walke \& Mamon 1989, for most HCGs). The galaxies in a chance alignment lie in a looser group (Mamon 1986;
Walke \& Mamon 1989), a cluster (Walke \& Mamon), or an even longer cosmological filament (Hernquist et al. 1995). Although HCG galaxies display numerous signs of dynamical interaction with close neighbors (Hickson 1997, and references therein), those HCGs caused by chance alignments are expected to be binary-rich (Mamon 1990, 1992), and these binaries should explain - to first order - the frequency of interacting galaxies (Mamon 1992).

Motivated by Walke \& Mamon's prediction that the frequency of chance alignments increases with the number of galaxies in the parent system, I had searched the Virgo cluster for CGs meeting Hickson's selection criterion, and indeed found a CG, composed of M60, M 59, NGC 4660, NGC 4638, and NGC 4647 (Mamon 1989). Figure 1 displays a view of this compact group (hereafter called the M 60 CG), taken from the Sloan Digital Sky Survey (SDSS).

The M 60 CG had been missed by Hickson (1982), because NGC 4606, an Sa galaxy lying at 1.98 Hickson circle radii from the group center, was only 2.4 mag fainter than M60 in the $B$ band. Even after a crude extrapolation to $R$ mag for the different morphological types of the two galaxies, NGC 4606 was still slightly less than 3 mag fainter than M 60, so NGC 4606 caused the CG to fail Hickson's isolation criterion. When I discovered this CG (Mamon 1989), I noticed that more accurate $B$-band photometry indicated that NGC 4606 was 2.88 mag fainter in $B$ 


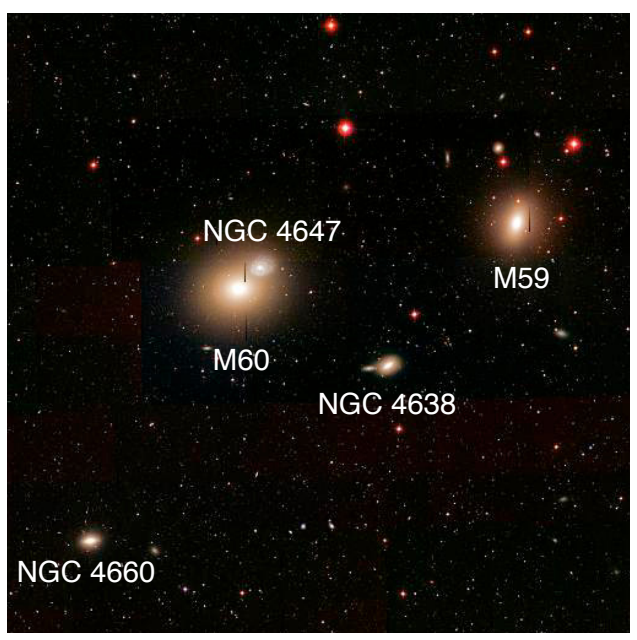

Fig. 1. SDSS mosaic of the M 60 compact group in RGB display using the $g, r$ and $i$ SDSS images. The image is 54'. 1 wide. The dark vertical lines in the Northern part of M 60 and the Western part of M 59 are image artefacts.

than M60, which, after the crude correction for morphological types, suggested that NGC 4606 was at least 3 mag fainter than M 60 in the $R$ band. I therefore argued that NGC 4606 did not affect the isolated status of the $\mathrm{CG}$, and concluded that the M 60 CG was the nearest HCG-like group.

The nature of CGs within clusters is by no means clear. While chance alignments are expected to be frequent in clusters (Walke \& Mamon 1989), one also expects to see groups falling in or bouncing out of clusters before becoming dynamically mixed with their host cluster. The tidal field of the cluster should truncate the infalling groups after their first passage, leaving groups with high density close to that of the cluster at pericenter (Mamon 1995; Gnedin et al. 1999), which is of the same order as the mean density of the M 60 CG.

Recent measurements by Mei et al. (2007) of the distances to Virgo elliptical and lenticular galaxies, through the accurate surface brightness fluctuation (SBF) method (Tonry \& Schneider 1988), permit to check whether the M 60 CG is dense in 3D or whether it is caused by a chance alignment of galaxies. This is the first CG meeting the HCG criteria that is sufficiently nearby to have its nature determined by SBF distance measurements.

In this Research Note, I first re-investigate in Sect. 2 whether the latest photometric measurements confirm that NGC 4606 is too faint to be considered a contaminant of the isolation ring. I then present briefly, in Sect. 3, the SBF distance measurements. In Sect. 4, I estimate lower limits for the line-of-sight separations of the M60 CG galaxies, given the SBF distance measurements and their errors, which I compare in Sect. 5 to the maximum line-of-sight size that I estimate is possible for truly dense groups. I also investigate which other HCGs are both close enough and with sufficient numbers of bright early-type galaxies to have their nature determined by SBF measurements with present-day accuracies.

\section{Is NGC 4606 sufficiently bright to affect the isolated status of the M 60 compact group?}

Figure 2 shows the large-scale environment of the M 60 CG, with NGC 4606 lying within the isolation ring. Is NGC 4606 bright enough to prevent the M 60 CG from being isolated? Following

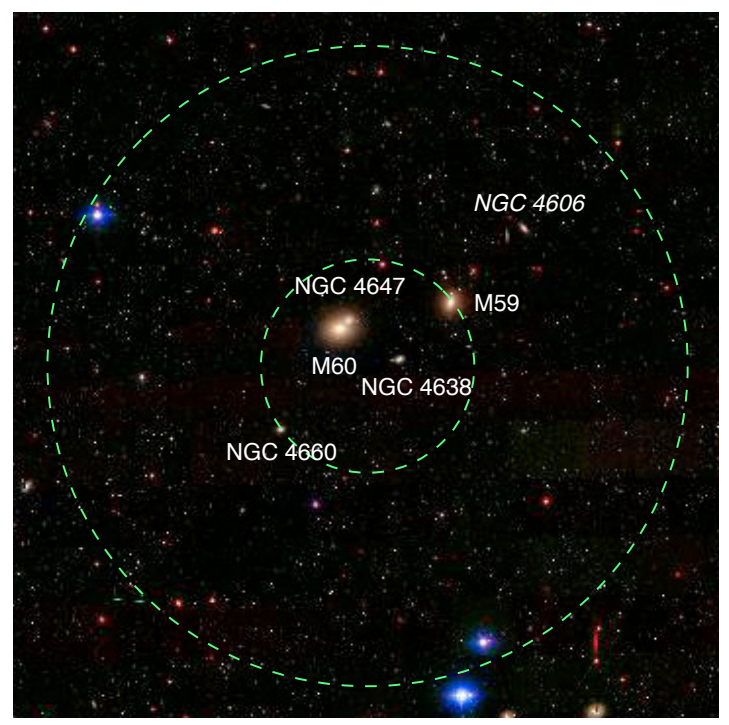

Fig. 2. SDSS mosaic of the M 60 compact group and its environment in RGB display using the $g, r$ and $i$ SDSS images. The image is 2.7 wide. The inner and outer circles show the limit of the group and the outer radius of the isolation ring, respectively. The two bright blue objects in the ring are foreground stars.

Hickson's original isolation criterion, the M $60 \mathrm{CG}$ is isolated if NGC 4606 is over 3 mag fainter than M 60 in the $R$ band.

Unfortunately, there is yet no good $R$-band photometry for NGC 4606 and M 60. The 6th Data Release of the Sloan Digital Sky Survey (SDSS) obtained photometric measurements for NGC 4606, but with bad photometric flags. As for measurements of other bright galaxies, the SDSS photometric measurement for M 60 is off by $\approx 3$ mag (Mamon et al., in prep.), probably because of poor background subtraction. For these reasons, neither galaxy has SDSS photometry in the NASA/IPAC Extragalactic Database (NED).

I attempted to measure the photometry of these two galaxies directly from the SDSS images. A SExtractor (Bertin \& Arnouts 1996) extraction of NGC 4606 (using large $512 \times 512$ pixel tiles to estimate the background, thus avoiding an overestimate of the background at the position of the large galaxy), gave $r=11.77 \pm 0.00$ (while its magnitude in the SDSS database is $r=12.22 \pm 0.00)$. On the other hand, M 60 is located near the edge of its $14^{\prime} \times 10^{\prime}$ scan, and its image almost fills the entire scan, so that the background subtraction is uncertain, which leads to important uncertainties in the photometry for M 60 (one can also distinguish different background levels in the SDSS mosaic of Fig. 1).

Figure 3 shows the difference in magnitude between NGC 4606 and the giant elliptical M60 for different wavelengths. Given its morphological type, NGC 4606 is bluer than M60 (as can be seen in Fig. 2). Fitting a cubic spline to the magnitude difference as a function of log wavelength, NGC 4606 is found to be 3.11 mag fainter than M60 in the $R$ band. However, assuming Gaussian-distributed magnitude errors, a simple Monte Carlo analysis (with $10^{5}$ trials) shows that only $81 \%$ of the time is the magnitude difference in the $R$ band greater than 3 mag.

Alternatively, the total $R$-band photometry of M60 and NGC 4606 can be found by extrapolating the $B$ or $V$ total photometry from the RC3 (de Vaucouleurs et al. 1991) using $B-R$ or $V-R$ colors measured in annuli at roughly half the 


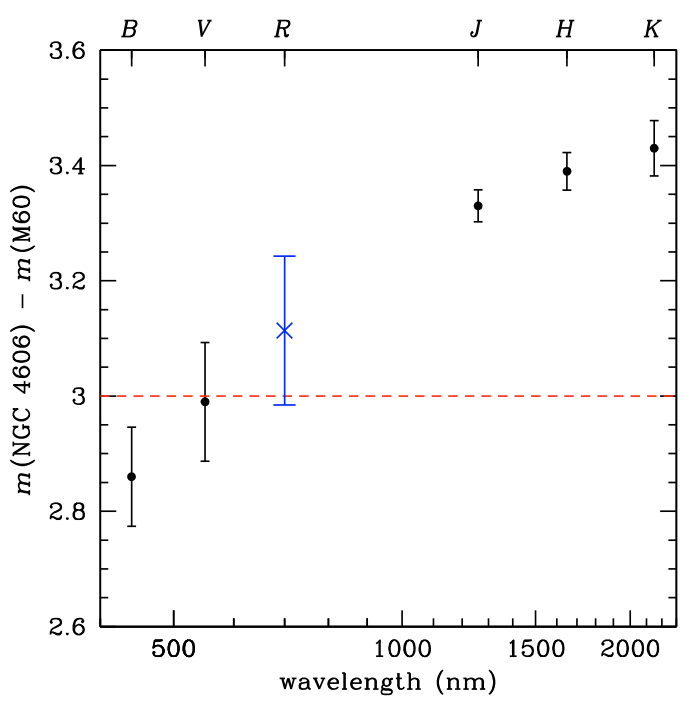

Fig. 3. Magnitude difference between NGC 4606 and M 60 in different wavebands: $B_{\mathrm{T}}, V_{\mathrm{T}}$ from the RC3 (de Vaucouleurs et al. 1991) and $J$, $H, K$ from 2MASS (Jarrett et al. 2000), all read in NED. The cross is the spline fitted value for the $R$ band (with Monte-Carlo $1 \sigma$ error).

luminosity. M 60 has $B_{\mathrm{T}}=9.81 \pm 0.05$ (de Vaucouleurs et al. 1991) and a color $B-R=1.59$ at the effective radius (from Peletier et al. 1990), yielding $R_{\mathrm{T}} \simeq 8.22 \pm 0.05$. NGC 4606 has $V_{\mathrm{T}}=11.83 \pm 0.09$ (de Vaucouleurs et al.) and $V-R=0.48$, which is the median of four measurements by Schröder \& Visvanathan (1996). This yields $R_{\mathrm{T}} \simeq 11.35 \pm 0.09$ for NGC 4606, hence the difference in $R$-band total magnitudes is $3.13 \pm 0.10$. For Gaussian-distributed errors, this leads to a $90 \%$ probability that NGC 4606 is too faint to destroy the isolation of the M 60 CG. The errors here do not include uncertainties in the colors nor in the calibration.

Hence, the M60 compact group has a good probability of being isolated (according to Hickson's criterion), but one cannot statistically rule out that NGC 4606 is bright enough in the $R$ band to spoil the group's isolation.

\section{SBF distance data}

As part of the ACS Virgo Cluster Survey (Côté et al. 2004), Mei et al. (2007) analyzed Hubble Space Telescope images to measure distances to 84 Virgo cluster ellipticals and S0s using the SBF method.

Mei et al. calibrated the SBF distances by fitting the trend of SBF apparent magnitude vs. $\left(g_{475}-z_{850}\right)_{0}$ color. They provided SBF distances using three different fits: linear, polynomial, and broken-linear. Mei et al. expressed their preference for the (4-parameter) broken-linear calibrated SBF distances. They noted that the $\chi^{2}$ of their broken-line and (4-parameter) 4th-order polynomial fits were equally good, while their linear (2 parameter) fit produced a slightly greater $\chi^{2}$. They also remarked that the broken-line fit had a smaller $\chi^{2}$ than the polynomial fit if the (three) galaxies redder than $\left(g_{475}-z_{850}\right)_{0}=1.5$ were excluded.

According to Table 2 of Mei et al., M 60 turns out to be the reddest (and 3rd brightest) galaxy in Virgo, with $\left(g_{475}-z_{850}\right)_{0}=$ 1.56. So, one infers that the polynomial calibration is superior for M 60, while the broken-line calibration is better for the three other galaxies of the M 60 CG. I thus also consider a mixed calibration which is broken-linear for $\left(g_{475}-z_{850}\right)_{0}<1.5$ and polynomial for $\left(g_{475}-z_{850}\right)_{0} \geq 1.5$ (the broken-linear and polynomial fits intersect at this critical color, so the mixed calibration is continuous).

\section{Analysis}

Table 1 shows the data for the 4 group members for which SBF distance measurements are available. Figure 4 illustrates the distances to the 4 ellipticals in the M 60 CG and to the three brightest Virgo galaxies (besides M 60): M 87, M 49 and M 86. M 60 and NGC 4638 appear to be located at roughly the same distance as the three luminous Virgo galaxies, M 87, M 49 and M 86. On the other hand, M 59 and NGC 4660, whose SBF distances are consistent (regardless of the calibration used) appear to lie roughly 2 Mpc closer to us.

Assuming Gaussian errors in the distance moduli, the distribution of the difference in distances of galaxies 1 and 2 with measured distance moduli $\mu_{1}$ and $\mu_{2}$ and uncertainties $\sigma_{1}$ and $\sigma_{2}$ is a Gaussian with mean $\mu_{2}-\mu_{1}$ and distribution $\sqrt{\sigma_{1}^{2}+\sigma_{2}^{2}}$. Hence, the probability that the difference in distance moduli of the two galaxies is greater than $\Delta \mu$ is

$P(\Delta \mu)=\frac{1}{2}\left\{1+\operatorname{erf}\left[\frac{\Delta \mu-\left(\mu_{2}-\mu_{1}\right)}{\sqrt{2\left(\sigma_{1}^{2}+\sigma_{2}^{2}\right)}}\right]\right\}$,

where $\operatorname{erf}(x)$ is the error function. Expressing the distance difference $\Delta D$ in terms of the difference in distance moduli $\Delta \mu$ as $\Delta D=2 D(\bar{\mu}) \sinh (0.1 \ln 10 \Delta \mu)$ and using Eq. (1), the minimum difference in distances of two galaxies is

$$
\begin{aligned}
(\Delta D)_{\min }= & 2 D(\bar{\mu}) \\
& \times \sinh \left\{\frac{\ln 10}{10}\left[2 \sigma_{\mathrm{rms}} \operatorname{erf}^{-1}(2 P-1)+\Delta \mu\right]\right\},
\end{aligned}
$$

where $y=\operatorname{erf}^{-1}(x)$ is the inverse error function, i.e. $\operatorname{erf}(y)=x$.

Table 2 provides the minimum distance difference between various pairs of galaxies of the M $60 \mathrm{CG}$, using Eq. (2). All three SBF estimators indicate that NGC 4638 is at least $800 \mathrm{kpc}$ more distant than M 59 and 1 Mpc more distant than NGC 4660 (both at the $99 \%$ confidence level). Moreover, using the broken-linear or linear SBF calibrations, M 60 must lie at least $0.82 \mathrm{Mpc}(99 \%$ confidence) further away than either M 59 or NGC 4660. On the other hand, the SBF distances determined with the polynomial or mixed calibrations produce consistent distances between M 60 and either M 59 or NGC 4660. However, one can combine the distances to M 59 and NGC 4660 to obtain a $\sqrt{2}$ smaller uncertainty in the distance of that galaxy pair. M 60 then turns out to be 440 or $520 \mathrm{kpc}$ further away than the pair (at the $95 \%$ confidence level), depending on which of the polynomial or brokenlinear SBF calibrations is used to estimate the distance of the pair.

\section{Discussion}

What is the maximum line-of-sight separation that is allowed for a galaxy pair located within a dense group of galaxies? Or equivalently, what is the maximum line-of-sight size of a dense group of galaxies?

One can specify that the maximum line-of-sight separation between galaxy pairs in a dense group must be smaller than twice its projected diameter or, alternatively, twice the 84th percentile (corresponding to $+1 \sigma$ for a Gaussian distribution) of the projected diameters of HCGs. Given that the angular radius 
Table 1. Data including SBF distance moduli to the galaxies in the M 60 compact group.

\begin{tabular}{|c|c|c|c|c|c|c|c|c|c|c|}
\hline \multicolumn{3}{|c|}{ Galaxy } & RA & Dec & \multirow[t]{2}{*}{ type } & \multirow[t]{2}{*}{$B_{\mathrm{T}}$} & \multirow{2}{*}{$\begin{array}{c}v \\
\left(\mathrm{~km} \mathrm{~s}^{-1}\right)\end{array}$} & \multicolumn{3}{|c|}{ distance modulus } \\
\hline Messier & NGC & $\mathrm{VCC}$ & & & & & & broken-line & linear & polynomial \\
\hline 59 & 4621 & 1903 & $12^{\mathrm{h}} 42^{\mathrm{m}} 02^{\mathrm{s}} \cdot 3$ & $+11^{\circ} 38^{\prime} 49^{\prime \prime}$ & E5 & 10.57 & 410 & $30.86 \pm 0.06$ & $30.92 \pm 0.03$ & $30.89 \pm 0.05$ \\
\hline - & 4638 & 1938 & $12^{\mathrm{h}} 42^{\mathrm{m}} 47^{\mathrm{s}} .4$ & $+11^{\circ} 26^{\prime} 33^{\prime \prime}$ & So & 12.13 & 1164 & $31.21 \pm 0.05$ & $31.15 \pm 0.04$ & $31.18 \pm 0.05$ \\
\hline - & 4647 & 1972 & $12^{\mathrm{h}} 43^{\mathrm{m}} 32^{\mathrm{s}} \cdot 3$ & $+11^{\circ} 34^{\prime} 55^{\prime \prime}$ & $\mathrm{Sc}$ & 11.94 & 1422 & - & - & - \\
\hline 60 & 4649 & 1978 & $12^{\mathrm{h}} 43^{\mathrm{m}} 40^{\mathrm{s}} \cdot 0$ & $+11^{\circ} 33^{\prime} 09^{\prime \prime}$ & E2 & 9.81 & 1117 & $31.19 \pm 0.07$ & $31.31 \pm 0.04$ & $31.06 \pm 0.06$ \\
\hline - & 4660 & 2000 & $12^{\mathrm{h}} 44^{\mathrm{m}} 32^{\mathrm{s}} .0$ & $+11^{\circ} 11^{\prime} 26^{\prime \prime}$ & E5 & 12.16 & 1083 & $30.88 \pm 0.05$ & $30.84 \pm 0.04$ & $30.88 \pm 0.05$ \\
\hline
\end{tabular}

Notes: positions, types and heliocentric velocities are from NED, magnitudes from RC3, and distance moduli from Mei et al. (2007).

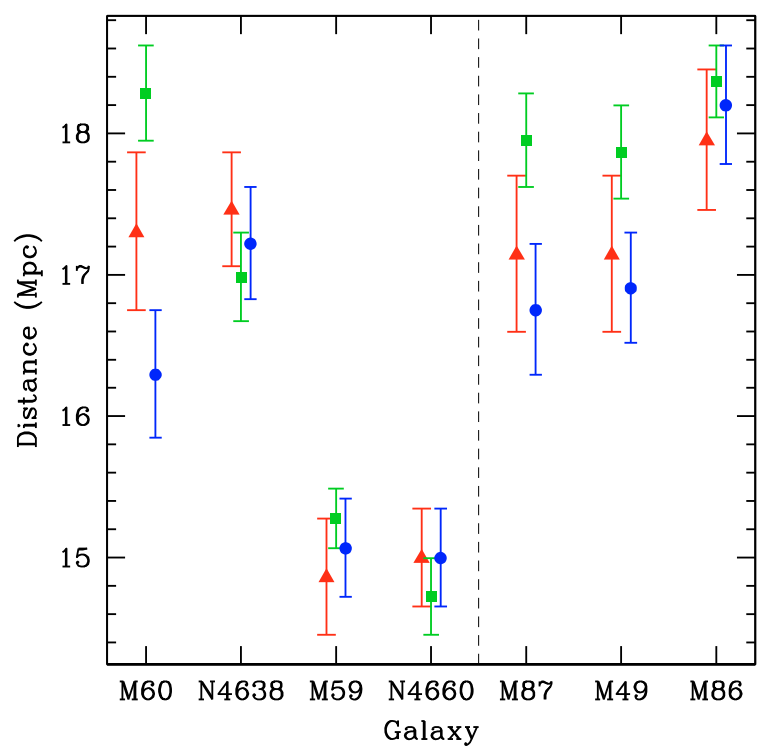

Fig. 4. Surface brightness fluctuation distances (from Mei et al. 2007) for the 4 ellipticals in the M 60 compact group (left) and for the three other brightest ellipticals in the cluster (right). The red triangles, green squares and blue circles represent the distance measurements using the broken-line, linear, and polynomial calibrations, respectively.

of the Hickson circle of the M 60 CG is 0.38 (Mamon 1989) ${ }^{1}$, and given its (error-weighted) mean (mixed SBF calibration) distance of $15.94 \mathrm{Mpc}$ (see Fig. 4), the projected radius of the Hickson circle is $106 \mathrm{kpc}$. In comparison, the median projected radius of the $68 \mathrm{HCGs}$ with at least 4 accordant velocities ${ }^{2}$ is $56 \mathrm{kpc}^{3}$, and $106 \mathrm{kpc}$ corresponds to the 87 th percentile of the distribution of HCG projected radii. Both criteria are therefore virtually identical. I thus adopt a maximum line-of-sight size of $4 \times 106=424 \mathrm{kpc}$.

According to Table 2, it is highly unlikely that NGC 4638 is part of a dense group or pair containing M59 and NGC 4660. Still, 4 galaxies remain once NGC 4638 is omitted. Nevertheless, M 60 cannot be part of the dense group or pair containing M 59 and NGC 4660, at a $99 \%$ (broken-linear or linear SBF calibrations) or $95 \%$ (polynomial SBF calibration) confidence level.

\footnotetext{
1 This angular radius is not affected by the removal of NGC 4647 (see Fig. 2).

2 I exclude HCG 54, the HCG with the smallest projected radius, because it does not constitute a group of galaxies, appearing instead to be either a group of H II regions in a single galaxy (Arkhipova et al. 1981) or the end result of the merger of two disk galaxies (Verdes-Montenegro et al. 2002).

${ }^{3}$ I assume $H_{0}=70 \mathrm{~km} \mathrm{~s}^{-1} \mathrm{Mpc}^{-1}$.
}

Table 2. Minimum line-of-sight separation of galaxy pairs.

\begin{tabular}{ccccc}
\hline \hline Galaxy 1 & Galaxy 2 & SBF calibration & \multicolumn{2}{c}{$\left(D_{2}-D_{1}\right)_{\min } /(1 \mathrm{Mpc})$} \\
\cline { 4 - 5 } & & & $P=0.95$ & $P=0.99$ \\
\hline M 59 & M 60 & broken-linear & 1.32 & 0.85 \\
M 59 & M 60 & polynomial & 0.30 & -0.08 \\
M 59 & M 60 & linear & 2.37 & 2.11 \\
M 59 & M 60 & mixed & $\mathbf{0 . 4 3}$ & $\mathbf{0 . 0 2}$ \\
\hline M 59 & NGC 4638 & broken-linear & $\mathbf{1 . 6 4}$ & $\mathbf{1 . 2 5}$ \\
M 59 & NGC 4638 & polynomial & 1.29 & 0.93 \\
M 59 & NGC 4638 & linear & 1.10 & 0.84 \\
\hline NGC 4660 & M 60 & broken-linear & 1.25 & 0.82 \\
NGC 4660 & M 60 & polynomial & 0.37 & -0.01 \\
NGC 4660 & M 60 & linear & 2.85 & 2.56 \\
NGC 4660 & M 60 & mixed & $\mathbf{0 . 3 7}$ & $\mathbf{- 0 . 0 1}$ \\
\hline NGC 4660 & NGC 4638 & broken-linear & $\mathbf{1 . 5 9}$ & $\mathbf{1 . 2 3}$ \\
NGC 4660 & NGC 4638 & polynomial & 1.36 & 1.00 \\
NGC 4660 & NGC 4638 & linear & 1.58 & 1.30 \\
\hline M 59+NGC 4660 & M60 & broken-linear & 1.39 & 0.99 \\
M 59+NGC 4660 & M 60 & polynomial & 0.44 & 0.09 \\
M 59+NGC 4660 & M 60 & linear & 2.69 & 2.44 \\
M 59+NGC 4660 & M 60 & mixed & $\mathbf{0 . 5 2}$ & $\mathbf{0 . 1 7}$ \\
\hline
\end{tabular}

Notes: the lines with mixed SBF calibrations use broken-line and polynomial SBF calibrations for Galaxy 1 and M60, respectively, which appear to be the most appropriate calibrations for those galaxies. For each combination of galaxies, the most suitable set of SBF calibrations are highlighted in bold.

Therefore, one can state with high confidence that among the four early-type galaxies in the M60 CG, M 59 and NGC 4660 cannot constitute a dense group of galaxies with M60 and NGC 4638.

The M 60 CG in Virgo is just one example of a Hickson-like compact group. Up to now, SBF distances have been measured for galaxies out to $v=4000 \mathrm{~km} \mathrm{~s}^{-1}$ (Tonry et al. 2001) and the ACS Virgo Cluster Survey has measured distances to galaxies as faint as $B_{\mathrm{T}}=16$ (Mei et al. 2007). There is one HCG within these distance and magnitude limits: HCG $68\left(v=2400 \mathrm{~km} \mathrm{~s}^{-1}\right.$, Hickson et al. 1992, 2 ellipticals and 2 S0s, all with $B_{\mathrm{T}} \leq 14.56$, Hickson et al. 1989, plus one Sbc), whose nature could therefore be probed in the same way as for the M60 CG. In the near future, SBF distances should become available for fainter and more distant early-type galaxies, allowing for direct line-of-sight analyses, similar to the present one, for additional HCGs.

Alternatively, the nature of the the full set of HCGs can be assessed by confronting the observational properties of these exceptionally dense galaxy systems with those constructed using either cosmological hydrodynamical simulations that can resolve sufficiently small galaxies, or alternatively, galaxy formation simulations based upon realistic galaxy positions 
obtained from high-resolution cosmological dark matter simulations. Using this second approach, McConnachie et al. (2008) have recently shown that roughly $70 \%$ of Hickson-like CGs selected in projection are chance alignments of galaxies, i.e. roughly half of those with at least 4 accordant velocities. Díaz-Giménez et al. (2008) find that this fraction depends on the cut-off in maximum line-of-sight size, and on the galaxy formation code ran on the outputs of the Millennium dark matter simulations (Springel et al. 2005).

Acknowledgements. The author thanks Simona Mei for several very useful discussions on the SBF distance indicator, Sandy Faber on SDSS photometry of very bright galaxies, and the anonymous referee for useful comments. This research has made use of the NASA/IPAC Extragalactic Database (NED) which is operated by the Jet Propulsion Laboratory, California Institute of Technology, under contract with the National Aeronautics and Space Administration, the HyperLEDA database (Paturel et al. 2003), the SDSS and GoogleSky (http://earth.google.com). Funding for the Sloan Digital Sky Survey (SDSS) and SDSS-II has been provided by the Alfred P. Sloan Foundation, the Participating Institutions, the National Science Foundation, the U.S. Department of Energy, the National Aeronautics and Space Administration, the Japanese Monbukagakusho, and the Max Planck Society, and the Higher Education Funding Council for England. The SDSS Web site is http: //www. sdss.org/. The SDSS is managed by the Astrophysical Research Consortium (ARC) for the Participating Institutions. The Participating Institutions are the American Museum of Natural History, Astrophysical Institute Potsdam, University of Basel, University of Cambridge, Case Western Reserve University, The University of Chicago, Drexel University, Fermilab, the Institute for Advanced Study, the Japan Participation Group, The Johns Hopkins University, the Joint Institute for Nuclear Astrophysics, the Kavli Institute for Particle Astrophysics and Cosmology, the Korean Scientist Group, the Chinese Academy of Sciences (LAMOST), Los Alamos National Laboratory, the Max-PlanckInstitute for Astronomy (MPIA), the Max-Planck-Institute for Astrophysics (MPA), New Mexico State University, Ohio State University, University of Pittsburgh, University of Portsmouth, Princeton University, the United States Naval Observatory, and the University of Washington.

\section{References}

Arkhipova, V. P., Afanasev, V. L., Dostal, V. A., et al. 1981, SvA, 25, 277 Bertin, E., \& Arnouts, S. 1996, A\&AS, 117, 393

Côté, P., Blakeslee, J. P., Ferrarese, L., et al. 2004, ApJS, 153, 223

de Vaucouleurs, G., de Vaucouleurs, A., Corwin, J. R., et al. 1991, Third Reference Catalogue of Bright Galaxies (New York: Springer)

Díaz-Giménez, E., Ragone-Figueroa, C., Muriel, H., \& Mamon, G. A. 2008, MNRAS, to be submitted

Gnedin, O. Y., Hernquist, L., \& Ostriker, J. P. 1999, ApJ, 514, 109

Hernquist, L., Katz, N., \& Weinberg, D. H. 1995, ApJ, 442, 57

Hickson, P. 1982, ApJ, 255, 382

Hickson, P. 1997, ARA\&A, 35, 357

Hickson, P., Kindl, E., \& Auman, J. R. 1989, ApJS, 7, 687

Hickson, P., Mendes de Oliveira, C., Huchra, J. P., \& Palumbo, G. G. 1992, ApJ, 399,353

Hickson, P., \& Rood, H. J. 1988, ApJ, 331, L69

Jarrett, T. H., Chester, T., Cutri, R., et al. 2000, AJ, 119, 2498

Mamon, G. A. 1986, ApJ, 307, 426

Mamon, G. A. 1989, A\&A, 219, 98

Mamon, G. A. 1990, in Paired and Interacting Galaxies, ed. J. W. Sulentic, W. C. Keel, \& J. Telesco (Washington: NASA), IAU Coll., 124, 619

Mamon, G. A. 1992, in Distribution of Matter in the Universe, ed. G. A. Mamon, \& D. Gerbal (Paris: Obs. de Paris), DAEC mtg., 2, 51

Mamon, G. A. 1995, in Third Paris cosmology colloquium, 95 [arXiv:astro-ph/9511101]

McConnachie, A. W., Ellison, S. L., \& Patton, D. R. 2008, MNRAS, in press [arXiv:0804.2928]

Mei, S., Blakeslee, J. P., Côté, P., et al. 2007, ApJ, 655, 144

Paturel, G., Petit, C., Prugniel, P., et al. 2003, A\&A, 412, 45

Peletier, R. F., Davies, R. L., Illingworth, G. D., Davis, L. E., \& Cawson, M. 1990, AJ, 100, 1091

Rose, J. A. 1977, ApJ, 211, 311

Schröder, A., \& Visvanathan, N. 1996, A\&AS, 118, 441

Springel, V., White, S. D. M., Jenkins, A., et al. 2005, Nature, 435, 629

Tonry, J., \& Schneider, D. P. 1988, AJ, 96, 807

Tonry, J. L., Dressler, A., Blakeslee, J. P., et al. 2001, ApJ, 546, 681

Verdes-Montenegro, L., Del Olmo, A., Iglesias-Páramo, J. I., et al. 2002, A\&A, 396,815

Walke, D. G., \& Mamon, G. A. 1989, A\&A, 225, 291 